\title{
The anti-tumor effect of miR-539-3p on colon cancer via regulating cell viability, motility, and nude mouse tumorigenicity with CDK14 inhibition
}

\author{
Zhuo Wang, Tao Hu, Chengwu Jin, Jiangui Yu, Dongqiang Zhu, Jian Liu \\ Department of General Surgery, Chengdu Fifth People's Hospital, Chengdu, China \\ Contributions: (I) Conception and design: All authors; (II) Administrative support: J Liu; (III) Provision of study materials or patients: All authors; (IV) \\ Collection and assembly of data: All authors; (V) Data analysis and interpretation: Z Wang, T Hu; (VI) Manuscript writing: All authors; (VII) Final \\ approval of manuscript: All authors. \\ Correspondence to: Jian Liu. Department of General Surgery, Chengdu Fifth People’s Hospital, Chengdu, China. Email: ndyrhrwik0@sina.com.
}

\begin{abstract}
Background: Colon cancer is one of the major causes of morbidity and mortality worldwide. MicroRNAs (miRNAs) play important functions in the growth and metastasis of colon cancer. This study aimed to investigate the anti-tumor effect of micro ribonucleic acid 539-3p (miR-539-3p) on colon cancer via regulation of cell viability, motility, and nude mouse tumorigenicity with cyclin-dependent kinase 14 (CDK14) inhibition.
\end{abstract}

Methods: The target relationship between miR-539-3p and CDK14 was predicted using TargetScan software, and were detected by luciferase reporter assay. Cell counting kit-8 (CCK-8) assay and flow cytometry were employed to examine cell proliferation and apoptosis. Western blotting was employed to measure the protein expression levels of p27, cleaved caspase-3, and epithelial (E)- and neural (N)-cadherin. The effect of miR-539-3p on tumor growth was evaluated by establishing a xenograft tumor model in nude mice.

Results: The target relationship of CDK14 and miR-539-3p was identified as a negative regulator. Overexpression of miR-539-3p significantly inhibited SW620 and SW480 cell proliferation, promoted cell apoptosis, and suppressed cell invasion by targeting CDK14. The xenograft tumor model showed that the overexpression of miR-539-3p reduced tumor weight and volume. Immunohistochemical staining revealed that the overexpression of miR-539-3p inhibited the expression of Ki67 and E-cadherin. Additionally, terminal deoxynucleotidyl transferase dUTP nick end labelling (TUNEL) staining showed that overexpression of miR-539-3p induced apoptosis.

Conclusions: Overexpression of miR-539-3p inhibited SW620 and SW480 cell proliferation, promoted cell apoptosis, and suppressed cell invasion by targeting CDK14. Therefore, miR-539-3p may be a useful diagnostic and therapeutic biomarker for colon cancer.

Keywords: Colon cancer; miR-539-3p; CDK14; proliferation; apoptosis; invasion

Submitted Aug 26, 2020. Accepted for publication Sep 29, 2020.

doi: 10.21037/jgo-20-387

View this article at: http://dx.doi.org/10.21037/jgo-20-387

\section{Introduction}

Colon cancer is one of the major causes of morbidity and mortality worldwide. It is usually treated by tumor resection, as chemotherapy and radiation have proven to be ineffective (1). Surgery is the primary treatment for localized colon cancer. However, diagnosis of the disease is often made at advanced stages due to the suboptimal efficiency of available diagnostic tools and the high metastatic potential of colon cancer. In this scenario, other therapies, such as chemotherapy and radiotherapy, 
are needed (2). Currently, combined treatment with fluoropyrimidine and oxaliplatin can improve the recurrence risk of patients undergoing resection of colon cancer. However, only about $20 \%$ of patients benefit from this adjuvant chemotherapy (3). Importantly, the toxicity of conventional chemotherapy drugs and their consequent adverse reactions have posed a significant challenge for clinicians (4). Dietary phytochemicals have been drawing increasing attention for colon cancer prevention and treatment due to their chemical diversity, biological activity, easy availability, lack of toxic effects, and ability to modulate various signal transduction pathways and cell processes (5), but there are lack of targeted treatment methods. Recently, micro ribonucleic acid (miRNA) targeted therapy for colon cancer has been extensively explored.

A miRNA is a short non-coding RNA molecule, and because it contains the core argonaute protein (AGO), it acts as a post-transcriptional regulator of gene expression (6). Numerous studies have reported that miRNA, as a tumor suppressor or tumor-promoting gene, plays a necessary role in inhibiting or promoting tumor cell drug resistance, apoptosis, proliferation, and invasion $(7,8)$. MicroRNA 539 (MiR-539) can inhibit cancer cell proliferation, migration, and invasion, and can also promote tumor cell apoptosis in breast (9), prostate (10), gastric (11) and pancreatic (12) cancers. MiR-539-3p inhibits proliferation and invasion of gastric cancer cells by targeting CTBP1 (13). MiR539-3p significantly promotes the proliferation, migration and invasion of SKOV3 cells. MiR-539-3p promotes the progression of epithelial ovarian cancer by targeting SPARCL1 (14). Importantly, miR-539 expression is downregulated in colorectal cancer tissues and cell lines, and is negatively correlated with an advanced clinical stage and lymph node metastasis (15).

In eukaryotic cells, cyclin-dependent kinases (CDKs) are key factors in cell cycle regulation, and their activation is regulated by related cell cycle proteins (16). Cyclindependent kinase 14 (CDK14), also known as PFTAIRE protein kinase 1 (PFTK1), is a novel member of the cell division cycle 2 (cdc2)-related serine/threonine protein kinases and CDK family (17). The oncogenic roles of CDK14 have been widely recognized in many studies. CDK14 is known to inhibit the Wingless-related integration site (Wnt) signaling pathway and accelerate the development of ovarian cancer (18). Moreover, low expression of dual-specificity tyrosine phosphorylation regulated kinase 2 (DYRK2) induces tumor cell proliferation and invasion by up-regulating CDK14 expression in breast cancer (19). CDK14 expression is also increased in esophageal cancer tissues and cell lines.

Furthermore, overexpression of CDK14 is positively correlated with clinicopathological variables such as tumor size, tumor grade, and survival rate. It also impedes the function of cisplatin chemotherapy by inducing esophageal squamous cell carcinoma proliferation (20). The CDK14 gene is identified as the target of miR-542-3p. Overexpression of CDK14 negatively regulates the inhibitory effect of miR-542-3p in epithelial ovarian cancer cell proliferation, invasiveness, and tumorigenesis (21). Interestingly, a comparison of the genome of the tumor, $\mathrm{CB} 42$, with the genomes of non-proliferative tumors via array comparative genomic hybridization (CGH) and sequencing reveals an amplicon on chromosome five containing CDK6 and CDK14, and a Kirsten rat sarcoma 2 viral oncogene homolog (KRAS) mutation in colon cancer (22).

Previous researches have displayed that miR-539 expression is down-regulated in colorectal cancer tissues and cell lines. However, the role of miR-539-3p in colon cancer has not been reported, and targeting relationship of between miR-539-3p and CD14K. And its function in cancer has not been reported. This study aims to evaluate whether the antitumor effect of miR-539-3p inhibits cell viability, motility, and tumorigenicity in nude mice by regulating CDK14 in colon cancer. We present the following article in accordance with the ARRIVE reporting checklist (available at http://dx.doi.org/10.21037/jgo-20-387).

\section{Methods}

\section{Cell culture}

Colon cancer cell lines (SW620 and SW480) used in this study were purchased from American Type Culture Collection (ATCC, Manassas, VA). All colon cancer cells were maintained and cultured separately in Eagle's Minimum Essential Medium (EMEM, Gibco, USA), supplemented with $10 \%$ fetal bovine serum (FBS, Life Technologies, Grand Island, USA). The cells were subsequently incubated at $37^{\circ} \mathrm{C}$ in a tissue culture chamber with $95 \%$ oxygen $\left(\mathrm{O}_{2}\right)$ and $5 \%$ carbon dioxide $\left(\mathrm{CO}_{2}\right)$.

\section{Cell transfection}

MicroRNA-negative control (miR-NC) micro ribonucleic acid 539-3p (miR-539-3p) mimic, plasmid cloning deoxyribonucleic acid (pcDNA), and pcDNA-CDK14 
Table 1 Primer sequences

\begin{tabular}{lcc}
\hline Gene & Forward primer & Reverse primer \\
\hline miR-539-3p & GCAGGAACGGTTCTCCACTC & GTCCCTAGGGATGTGTAAGGA \\
CDK14 & CAAACCCCTGGACACAATTCCTG & CGAGCTGGGGCTGGAGTGCCG \\
E-cadherin & CGAGAGCTACACGTTCACGG & GGGTGTCGAGGGAAAAATAGG \\
N-cadherin & TTGATGGAGGTCTCCTAACACC & ACGTTTAACACGTTGGAAATGTG \\
\hline
\end{tabular}

were obtained from GenePharma (Shanghai, China). Lipofectamine 2000 (Invitrogen; Thermo Fisher Scientific, Inc., Waltham, MA, USA), a common transfection reagent, was employed to efficiently transfect miR-NC, miR-5393 p mimic, pcDNA, and pcDNA-CDK14 into SW620 and SW480 cells according to the manufacturer's instructions.

\section{Quantitative real-time polymerase chain reaction (qRT-PCR)}

Total RNA in SW620 and SW480 cells was isolated using the TRIzol reagent kit (Invitrogen, Beijing, China) according to the manufacturer's instructions. The total RNA concentration was measured using the GeneQuant Pro RNA/DNA Calculator (Amersham Pharmacia Biotec, UK). The PrimeScript RT reagent Kit (TakaRa, Dalian, China) was used to perform reverse transcription. SYBR Premix Ex Taq ${ }^{\text {TM }}$ II (TakaRa, Dalian, China) was used to assemble the qRT-PCR system, which was carried out in the Bio-Rad CFX-96 (Bio-Rad, CA, USA). Glyceraldehyde 3-phosphate dehydrogenase (GAPDH) was used for normalizing. The qRT-PCR data were analyzed using the $2^{-\Delta \Delta \mathrm{Ct}}$ method to calculate the relative expression levels of messenger RNA (mRNA). The primer sequences were used in Table 1.

\section{Western blot assay}

A radioimmunoprecipitation assay (RIPA) lysis buffer (Beyotime Institute of Biotechnology, Shanghai, China) was used to extract proteins in SW620 and SW480 cells according to the manufacturer's protocol. A bicinchoninic acid (BCA) protein assay kit was used to measure the protein concentrations. Primary antibody rabbit anti-p27 antibody (1:1,000, \#3686, Cell Signaling), anti-cleaved caspase-3 (1:1,000, \#9661, Cell Signaling), anti-E-cadherin (1:1,000, \#3195, Cell Signaling), and anti-N-cadherin $(1: 1,000, \# 13116$, Cell Signaling) were integrated with the target protein and incubated at room temperature for $1 \mathrm{~h}$. After that, the membranes were incubated with goat antirabbit immunoglobulin $\mathrm{G}$ (IgG) horseradish peroxidase (HRP)-conjugated secondary antibodies for $1 \mathrm{~h}$. The band densities were determined and analyzed using the Bio-Rad CFX-96 automatic digital gel image analysis system (BioRad, CA, USA).

\section{Dual luciferase reporter assay}

SW620 and SW480 cells were transfected with miR-539$3 p$ mimic and subsequently co-transfected CDK14-wild type (wt) or CDK14-mutant (mut) using Lipofectamine 2000. The dual-luciferase reporter assay system (Promega Corporation, Madison, WI, USA) was employed to analyze the luciferase assay. After $48 \mathrm{~h}$, the cells were collected and examined for luciferase activity as previously described (23).

\section{Cell viability (CCK-8) assays}

Cell viability was evaluated using a cell counting kit8 (CCK-8, Dojindo Molecular Technologies, Inc., Kumamoto, Japan) in both SW620 and SW480 cells according to the manufacturer's instructions. Finally, the absorbance was read using a microplate reader (Bio-Rad, California, USA) at $450 \mathrm{~nm}$.

\section{Flow cytometry analysis}

First, $3 \times 10^{6} / \mathrm{mL}$ suspended cells were collected into a $10 \mathrm{~mL}$ centrifuge tube, centrifuged at 100-200 g for $5 \mathrm{~min}$, and the culture solution was discarded. Next, the cells were washed once with incubation buffer, centrifuged at 100-200 $\mathrm{g}$ for $5 \mathrm{~min}$, and resuspended with $100 \mu \mathrm{L}$ of labeling solution, and then incubated at room temperature in the dark for $10-15 \mathrm{~min}$. The cells were subsequently centrifuged at 100-200 $\mathrm{g}$ for 5 min and washed again with the cell incubation buffer. Fluorescent (SA-FLOUS) solution was added, followed 
by incubation at $4{ }^{\circ} \mathrm{C}$ for $20 \mathrm{~min}$, avoiding light and with occasional vibration. Finally, flow cytometry analysis was performed with an excitation wavelength of $488 \mathrm{~nm}$, a bandpass filter with a wavelength of $515 \mathrm{~nm}$ for detecting fluorescein isothiocyanate (FITC) fluorescence, and another filter with a wavelength $>560 \mathrm{~nm}$ for detecting propidium iodide (PI). The Annexin V-FITC apoptosis detection kit $(\mathrm{C} 1062 \mathrm{~S})$ was purchased from the Shanghai Biyuntian Biotechnology Institute. Data analysis was performed using FlowJo (Tree Star, OR).

\section{The xenograft tumor model assay}

Animal experiments were conducted according to the National Institutes of Health (NIH) Guidelines for the Care and Use of Laboratory Animals and were approved by the Chengdu Fifth People's Hospital. All animals were raised in a specific pathogen free (SPF) environment and offered unlimited access to water and food. To test the effects of miR-539-3p on tumor growth, $5.0 \times 10^{6} \mathrm{SW620}$ cells were injected into the right flank near the hind leg of each nude mouse [Bagg Albino (BALB/c) athymic nude mice, female, 5-6 weeks-old, weight: $17.12 \pm 2.95 \mathrm{~g}$, purchased from Chengdu Dashuo Biotechnology Co.]. The experiments began when the mice bore palpable tumors (tumor volume of approximately $100 \mathrm{~mm}^{3}$ ). The mice were randomly divided into two groups: (I) transfection control group and (II) miR-539-3p mimic group.

\section{Immunobistochemistry}

Formalin-fixed, paraffin-embedded tissue sections were deparaffinized in xylene and rehydrated with gradient concentrations of ethanol. The tissue sections were stained with specific rabbit anti-Ki67 antibody (1:25, ab833, Abcam) and anti-N-cadherin (1:125, \#13116, Cell Signaling). The corresponding second antibody was incubated at room temperature for 1 hour. Finally, sections were observed using an Olympus DX51 fluorescence microscope (Olympus, Tokyo, Japan) and analyzed using Image6.0 software (Media Cybernetics, USA).

\section{Statistical analysis}

Each experiment in this study was carried out three times. The data were presented as mean \pm standard deviation (SD). Statistical analyses between two groups were conducted using the Student's $t$-tests and SPSS 25.0 software (IBM,
Armonk, USA). The significance of differences between treatment groups was examined using one-way analysis of variance (ANOVA). A P value $<0.05$ was considered statistically significant.

\section{Results}

\section{CDK14 is a target of miR-539-3p}

MiR-539 is down-regulated in many types of tumors, including colorectal cancer tissues and cell lines (15). CDK14 is up-regulated in many types of tumors, and is highly expressed in colon cancer cell lines (24). miRnegative control (NC) and miR-539-3p mimic were transfected into SW620 and SW480 cells. The results showed that the mRNA expression levels of miR-539$3 \mathrm{p}$ were up-regulated in the miR-539-3p mimic group compared with the control group in SW620 and SW480 cells (Figure $1 A, \mathrm{P}<0.05$ ). However, CDK14 was downregulated in the miR-539-3p mimic group compared with the control group in SW620 and SW480 cells (Figure 1B, $\mathrm{P}<0.05)$. Pc-DNA and pcDNA-CDK14 were transfected into SW620 or SW480 cells. The mRNA expression levels of CDK14 were elevated in the pcDNA-CDK14 group compared with the control group in both SW620 and SW480 cells (Figure $1 C, \mathrm{P}<0.05$ ). The potential targets of miR-539-3p and CDK14 were searched by using bioinformatics algorithms (Figure 1D). The dual-luciferase reporter assay was carried out on SW620 and SW480 cells; luciferase activity was inhibited significantly in SW620 and SW480 cells co-transfected with the CDK14 3'-UTR wt and miR-539-3p (Figure 1E,F). These results suggested that miR-539-3p could negatively regulate the expression of CDK14 via interaction with the three prime untranslated region (3'-UTR) of CDK14 mRNA.

\section{miR-539-3p inbibited cell proliferation by targeting CDK14 in vitro}

MiR-NC, miR-539-3p mimic, pcDNA-CDK14, and miR539-3p + CDK14 were transfected into SW620 and SW480 cells. The results indicated that the protein expression levels of CDK14 were decreased in the miR-539-3p group compared with the control group, but were elevated in SW620 and SW480 cells. However, the levels of CDK14 were decreased in the miR-539-3p + CDK14 group compared with the CDK14 group (Figure $2 A, \mathrm{P}<0.05$ ). SW620 and SW480 cell proliferation was detected using 

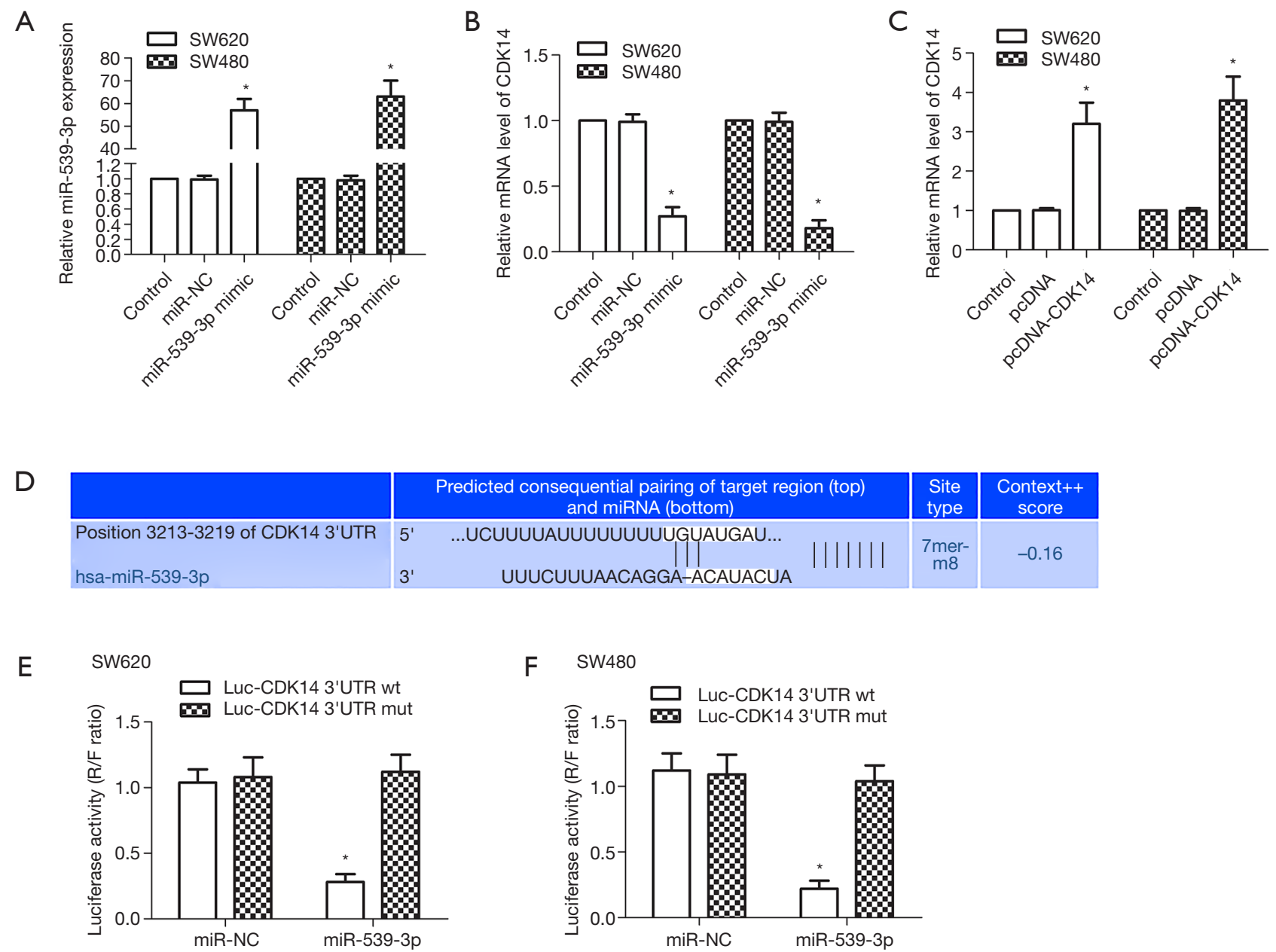

Figure 1 CDK14 is a target of miR-539-3p. (A) The mRNA expression of miR-539-3p was detected by qRT-PCR in both SW620 and SW480 cells in the control, miR-NC, and miR-539-3p mimic groups; (B) the mRNA expression of CDK14 was detected by qRT-PCR in both SW620 and SW480 cells in the control, miR-NC, and miR-539-3p mimic groups; (C) the mRNA expression of CDK14 was detected by qRT-PCR in both SW620 and SW480 cells in the control, pcDNA, and pcDNA-CDK14 groups; (D) prediction of binding sites between CDK14 and miR-539-3p; (E) Luciferase activity was presented relative to that of control and mutant or wild-type CDK14 3'-UTR in SW620 cells; (F) Luciferase activity was presented relative to that of control and mutant or wild-type CDK14 3'-UTR in SW480 cells. *, $\mathrm{P}<0.05$ compared with control group.

CCK-8. Both experiments showed that the up-regulation of CDK14 induced SW620 and SW480 cell proliferation. Meanwhile, the down-regulation of CDK14 suppressed SW620 and SW480 cell proliferation (Figure 2B). The protein expressive levels of Ki67 and PCNA were inhibited in the miR-539-3p group compared with control group, but the levels of Ki67 and PCNA were increased in the CDK14 group. However, the levels of Ki67 and PCNA were increased in the miR-539-3p + CDK14 group compared with CDK14 group (Figure 2C). These results indicated that
miR-539-3p could inhibit the cell proliferation by targeting CDK14.

\section{miR-539-3p promoted cell apoptosis by targeting CDK14 in vitro}

Flow cytometry demonstrated that SW620 and SW480 cell apoptosis was enhanced in the miR-539-3p group, and were restrained in the CDK14 group compared with the control group. However, SW620 and SW480 cells 
A
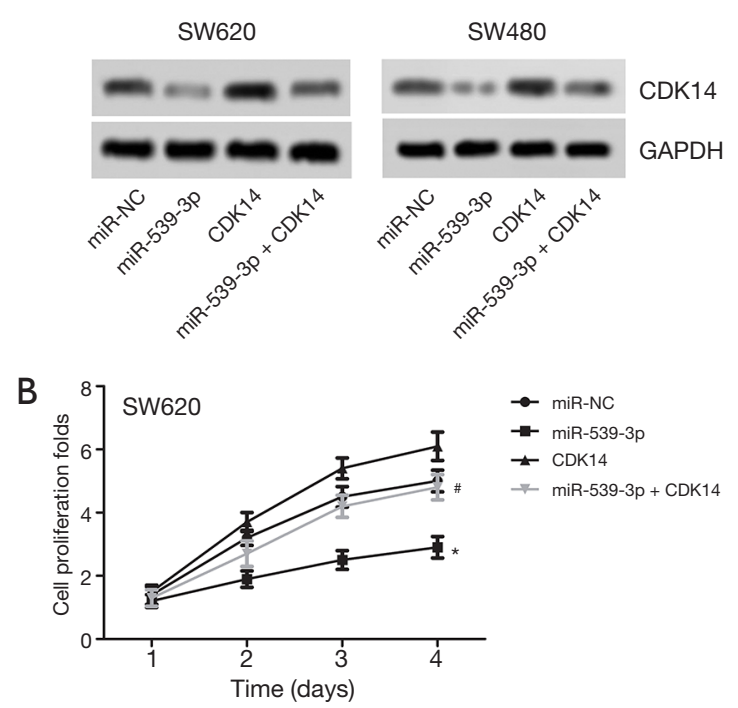

C SW620

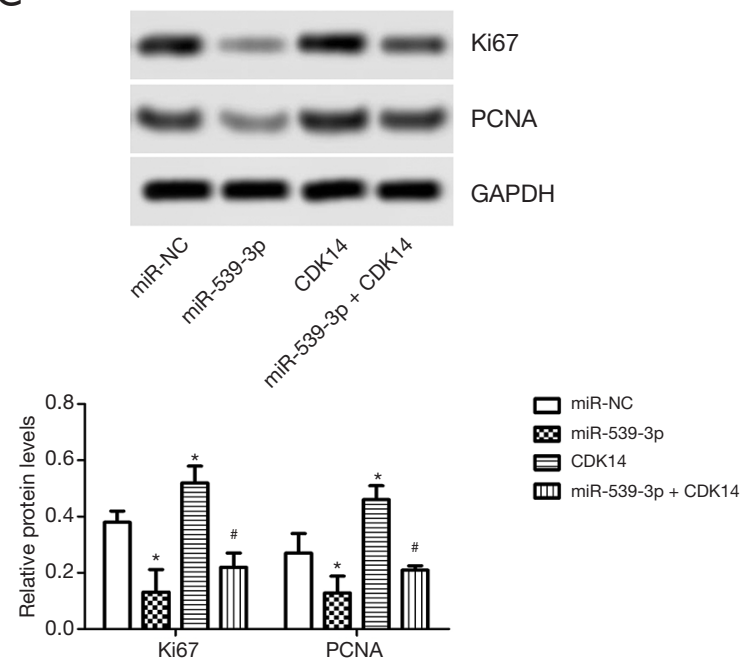

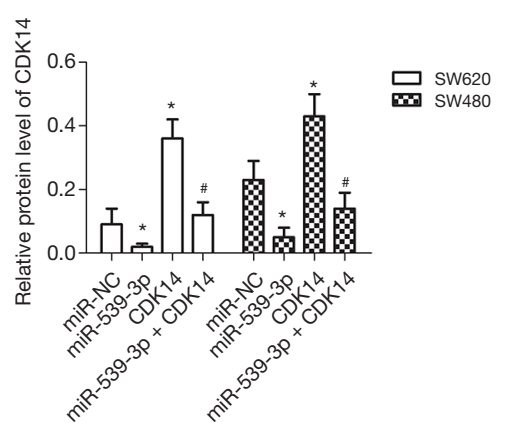

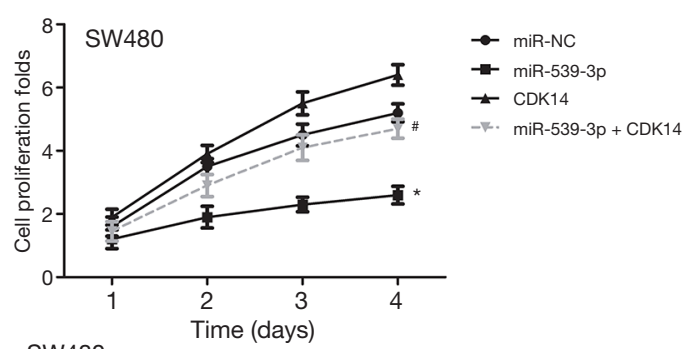

SW480
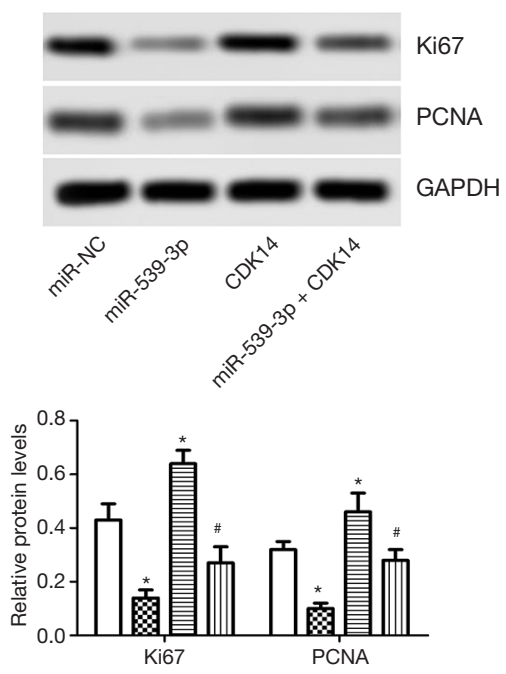

Figure 2 MiR-539-3p inhibited cell proliferation by targeting CDK14 in vitro. (A) The relative protein levels of CDK14 were detected by western blot in both SW620 and SW480 cells. Semi-quantitative analysis of the relative protein levels of CDK14 in the control, miR-5393p, CDK14, and miR-539-3p+ CDK14 groups. (B) The proliferation ability of both SW620 and SW480 were determined byCCK-8 assay. (C) The protein expression levels of Ki67 and PCNA were detected by western blot. *, $\mathrm{P}<0.05$ compared with control group; ${ }^{*}, \mathrm{P}<0.05$ compared with CDK14 group.

apoptosis was enhanced in the miR-539-3p + CDK14 group compared with the CDK14 group (Figure $3 A, \mathrm{P}<0.05$ ). The protein expression levels of p27 and cleaved caspase- 3 were measured by western blot. The results showed that the levels of p27 and cleaved caspase- 3 were elevated in the miR-539-3p group, and were inhibited in the CDK14 group compared with the control group in both SW620 and SW480 cells. However, the levels of p27 and cleaved caspase-3 were enhanced in the miR-539-3p + CDK14 group compared with the CDK14 group (Figure $3 B$, $\mathrm{P}<0.05)$. These results indicated that miR-539-3p could promote cell apoptosis by targeting CDK14.

\section{miR-539-3p inbibited cell invasion by targeting CDK14 in vitro}

The mRNA and protein expression levels of E- and $\mathrm{N}$-cadherin were measured by qRT-PCR and western 

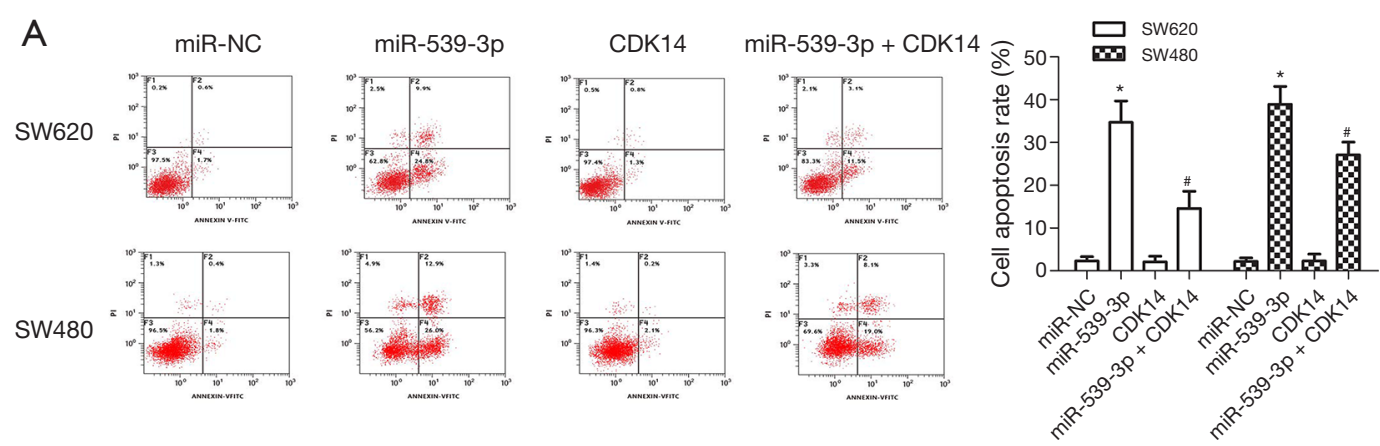

B
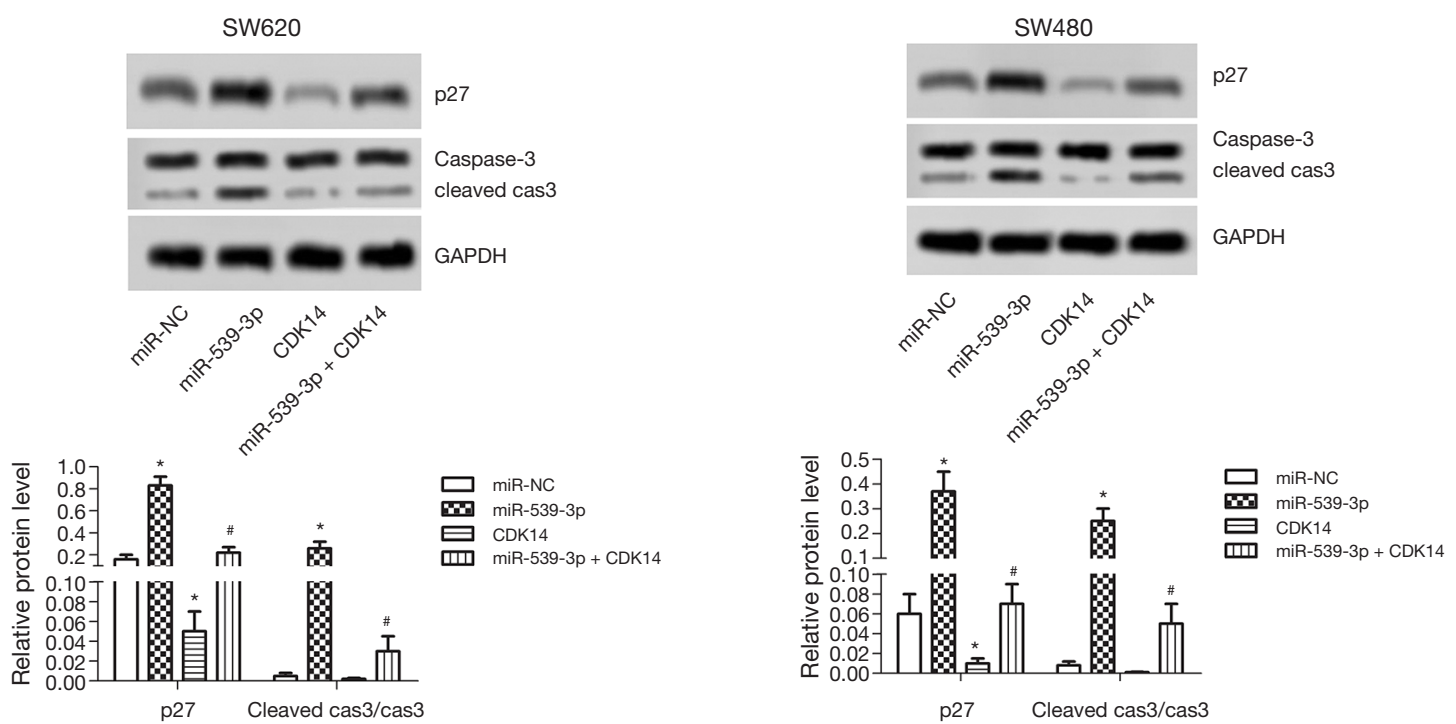

Figure 3 MiR-539-3p promoted cell apoptosis by targeting CDK14 in vitro. (A) Both SW620 and SW480 cells apoptosis were detected by flowcytometry; (B) the protein expression levels of p27 and cleaved caspase-3 were detected by western blot. ${ }^{*}$, $\mathrm{P}<0.05$ compared with control group; ${ }^{\#}, \mathrm{P}<0.05$, compared with $\mathrm{CDK} 14$ group.

blot. The result showed that the levels of E-cadherin were elevated in the miR-539-3p group, and were inhibited in the CDK14 group compared with the control group in both SW620 and SW480 cells. However, the levels of E-cadherin were enhanced in the miR-539-3p + CDK14 group compared with the CDK14 group (Figure $4 A, B$, $\mathrm{P}<0.05)$. Furthermore, the results showed that the levels of $\mathrm{N}$-cadherin were inhibited in the miR-539-3p group, and were enhanced in the CDK14 group compared with the control group in both SW620 and SW480 cells. However, the levels of $\mathrm{N}$-cadherin were suppressed in the miR-539$3 \mathrm{p}+$ CDK14 group compared with the CDK14 group (Figure $4 A, B, \mathrm{P}<0.05$ ). These results indicated that miR539-3p could inhibit cell invasion by targeting CDK14.

\section{miR-539-3p inhibited tumor growth in vivo}

Tumor growth in the xenograft mouse model was reduced in vivo compared with the non-transfection miR-539-3p group. Similarly, xenograft tumor volume was lower in vivo compared with the non-transfection miR-539-3p group (Figure $5 A, B, \mathrm{P}<0.05$ ). In tumor tissues, the expression level of miR-539-3p was increased compared with the non-transfection miR-539-3p group, while the expression level of CDK14 was decreased (Figure 5C,D). Terminal deoxynucleotidyl transferase dUTP nick end labelling (TUNEL) staining showed that cell apoptosis increased in vivo compared with the non-transfection miR-539-3p group (Figure 5E, $\mathrm{P}<0.05)$. Markers of cell proliferation (Ki67) and invasion (E-cadherin) were significantly down-regulated 
A

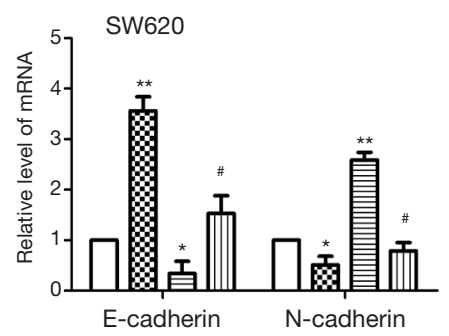

SW620

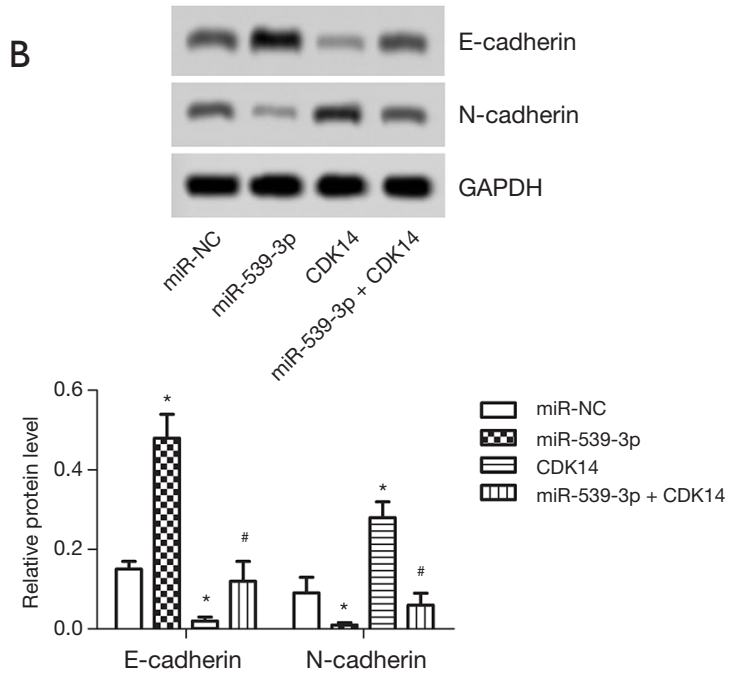

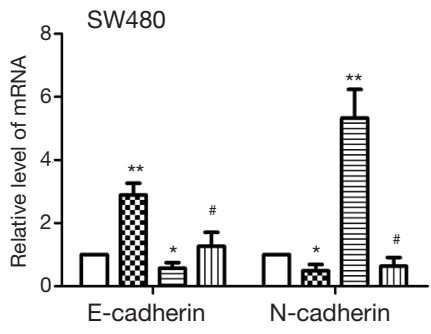

$$
\begin{aligned}
& \square \text { miR-NC } \\
& \text { miR-539-3p } \\
& \text { 曰 CDK14 } \\
& \text { miR-539-3p + CDK14 }
\end{aligned}
$$

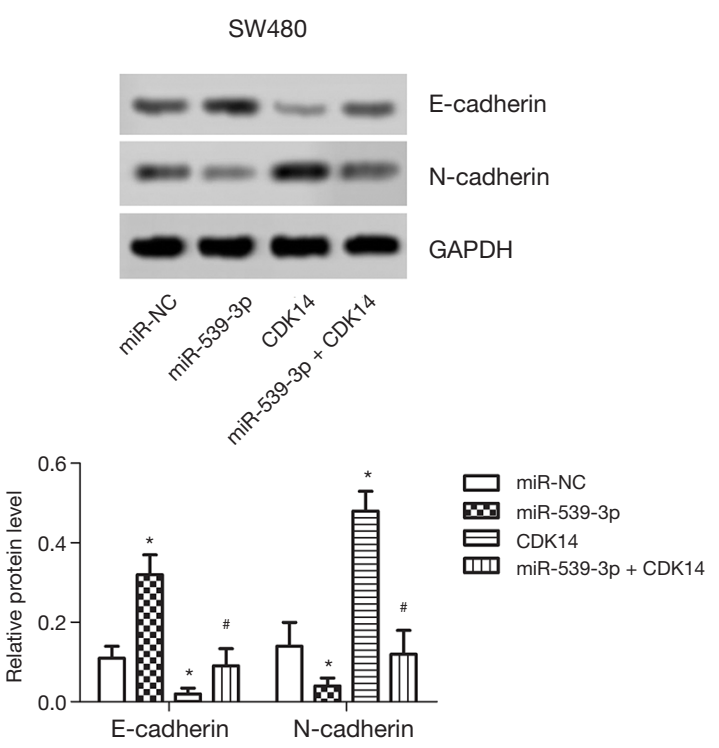

Figure 4 MiR-539-3p inhibited cell invasion by targeting CDK14 in vitro. (A) The mRNA expression levels of E- and N-cadherin were detected by qRT-PCR; (B) the protein expression levels of $\mathrm{E}$ - and $\mathrm{N}$-cadherin were detected by western blot. ${ }^{*}, \mathrm{P}<0.05 ;{ }^{* *}, \mathrm{P}<0.01$ compared with control group; ${ }^{\#}, \mathrm{P}<0.05$ compared with $\mathrm{CDK} 14$ group.

in vivo compared with the non-transfection miR-539-3p group (Figure $5 F, G, \mathrm{P}<0.05$ ). These results confirmed that up-regulation of miR-539-3p could inhibit tumor growth in vivo.

\section{Discussion}

P27 plays important roles in cell proliferation, differentiation, and apoptosis. It binds to and suppresses CDK to impede the cell cycle process and adjusts some processes, such as cell migration and development, independent of CDK (25). Previous studies have reported that p27 suppresses CDK6/cyclin D1 (CCND1) complex formation leading to cell cycle arrest and suppression of cell proliferation in lung cancer (26). It has also been widely recognized that caspase- 3 exerts a major executioner protein in proteolytic degradation during apoptosis. It can also regulate cell migration, invasion, and metastasis in colon cancer (27). In the present study, the levels of p27 and cleaved caspase- 3 were elevated in the miR-539-3p group, and were inhibited in the CDK14 group compared with the control group in both SW620 and SW480 cells. However, the levels of p27 and cleaved caspase- 3 were enhanced in the miR-539-3p + CDK14 group compared with the CDK14 group.

Epithelial-mesenchymal transition (EMT) is a typical phenomenon in cancer cells. In the process of EMT, polarized epithelial cells complete multifaceted changes that induce them to begin expressing a mesenchymal phenotype and undergo migration, invasion, and metastasis (28). E-cadherin mediates cell-cell adhesions and integrin mediates cell-matrix contacts, which regulates tumor invasion and metastasis (29). Lower expression of E-cadherin leads to loss of contact inhibition and is 
A

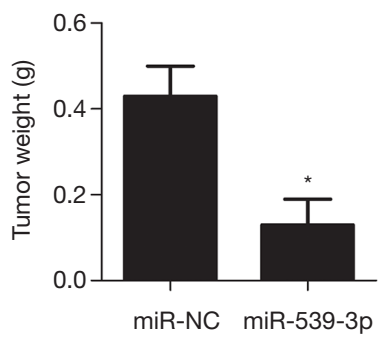

C

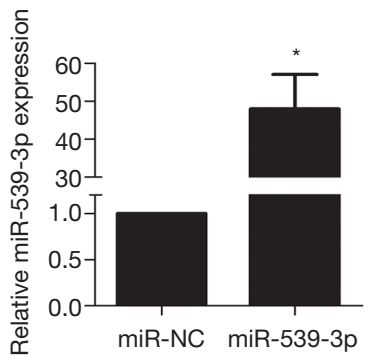

B
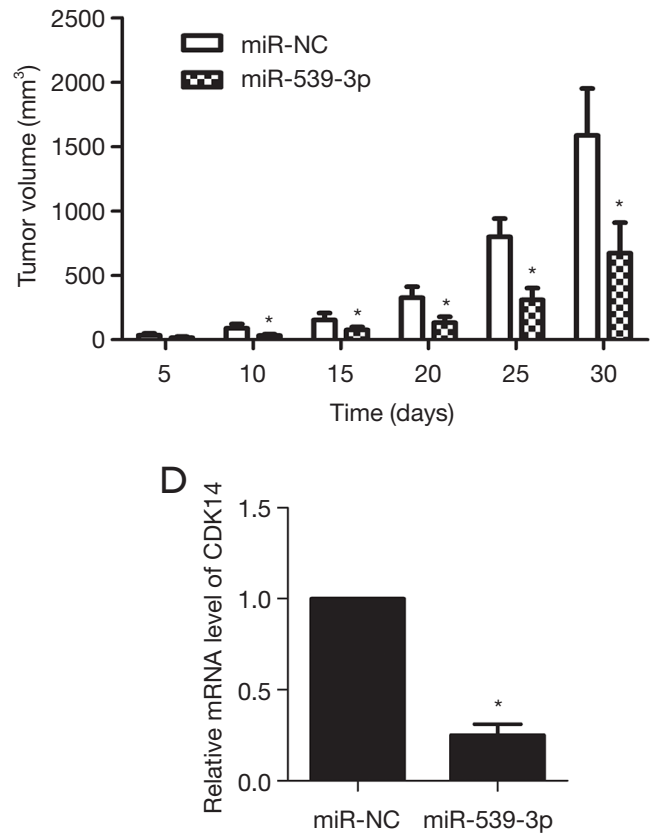
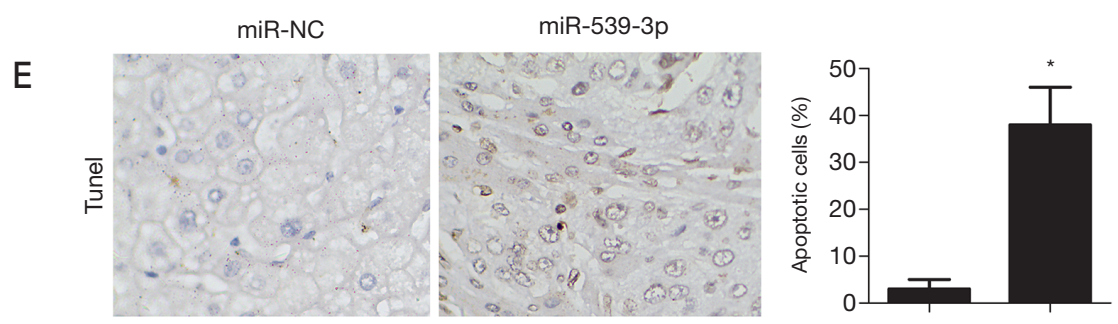

F
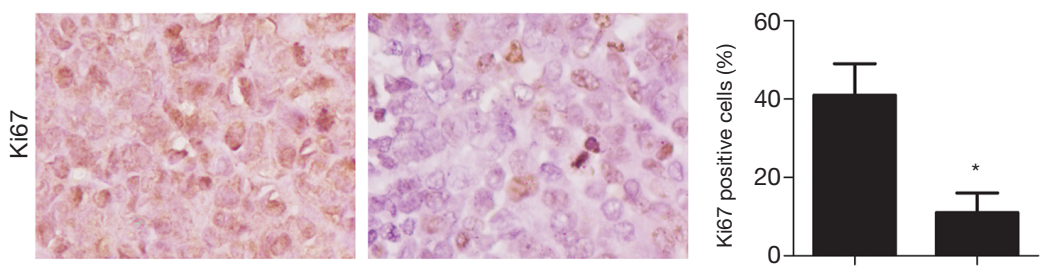

G
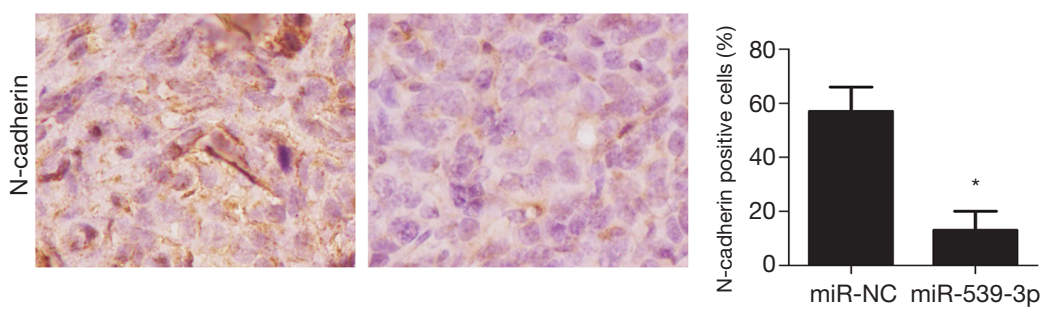

Figure 5 MiR-539-3p inhibited tumor growth in vivo. The effect of miR-539-3p on tumor growth was evaluated by establishing a xenograft tumor model. The mice was divided into a control group and a miR-539-3p mimic group. The histogram shows (A) tumor weight (g) and (B) tumor volume $\left(\mathrm{mm}^{3}\right)$. The mRNA expression levels of miR-539-3p (C) and CDK14 (D) were detected by qRT-PCR. (E) Cells apoptosis were detected by TUNEL straining (magnification $\times 400$ ). Immunohistochemistry analysis was employed to assess the expression of Ki67 (F) and E-cadherin $(\mathrm{G})($ magnification $\times 400)$. The histogram shows cell apoptosis rates, and the Ki67 and E-cadherin levels. * $\mathrm{P}<0.05$ compared with control group. 
associated with enhanced cell motility and advanced cancer (30). Also, N-cadherin functions as an invasion suppressor and is up-regulated in most carcinomas $(31,32)$. In the present study, the mRNA and protein expression levels of E- and N-cadherin were measured by qRT-PCR and western blot. The result showed that the levels of E-cadherin were elevated in the miR-539-3p group, and were inhibited in the CDK14 group compared with the control group in both SW620 and SW480 cells. However, the levels of E-cadherin were enhanced in the miR-539$3 p+$ CDK14 group compared with the CDK14 group. Moreover, we found that the levels of $\mathrm{N}$-cadherin were inhibited in the miR-539-3p group, and were enhanced in the CDK14 group compared with the control group in both SW620 and SW480 cells. However, the levels of N-cadherin were suppressed in the miR-539-3p + CDK14 group compared with the CDK14 group.

Numerous studies have reported that aberrant expression of miRNAs is correlated with cell proliferation, apoptosis, invasion, metastasis, drug resistance, and prognosis in various cancers $(33,34)$. Down-regulation of miR-539 is closely related to the National Wilms Tumor Study (NWTS)-5 stage, lymph node metastasis, and histological type in Wilms' Tumor (WT) patients. Moreover, there are correlations between the low expression of miR-539 and shorter overall survival rate in WT patients (35). Upregulation of miR-539 also inhibits tumor growth and tumorigenesis and prevents resistance to arsenic trioxide in hepatocellular carcinoma (36). In our research, upregulation of miR-539-3p inhibited SW620 and SW480 cell proliferation and invasion, and promoted cell apoptosis. In tumor tissues, we found that the expression level of miR539-3p was increased compared with the control group, while CDK14 expression was decreased. TUNEL staining showed that cell apoptosis was higher in vivo. Markers of cell proliferation (Ki67) and invasion (E-cadherin) were significantly down-regulated in vivo.

The oncogenic functions of CDK14 have been widely established. Overexpression of CDK14 indicates a poor prognosis in colorectal cancer (37). Importantly, the tumorigenesis-related cellular character, including invasiveness and motility, is reportedly correlated with the overexpression of CDK14 (38). Up-regulation of CDK14 also induces cell proliferation, migration, and invasion in breast cancer (39). Notably, knockdown of CDK14 inhibits colon cancer cell proliferation and invasion with EMT by suppressing the Sonic hedgehog signaling pathway (24). In our study, up-regulation of CDK14 induced SW620 and
SW480 cell proliferation and invasion, and inhibited cell apoptosis.

MiR-539 reduces resistance to cisplatin by directly targeting doublecortin-like kinase 1 (DCLK1) in nonsmall cell lung cancer cells (40). MiR-539 also has tumorsuppressive functions in colorectal cancer during the process of malignant transformation by directly targeting the SRY box transcription factor 4 (SOX4) (41). In pancreatic cancer, miR-431 suppresses cell proliferation and promotes cell apoptosis by down-regulating CDK14 (42). Moreover, miR-613 restrains glioma cell proliferation and invasion by down-regulating CDK14 (43). In this study, we found that CDK14 is a target of miR-539-3p. At the same time, miR539-3p could inhibit SW620 and SW480 cell proliferation and invasion, and accelerate apoptosis by targeting CDK14.

In conclusion, CDK14 is a target of miR-539-3p. MiR-539-3p was found to inhibit SW620 and SW480 cell proliferation and invasion, and accelerate apoptosis bytargetingCDK14. Our results confirmed that upregulation of miR-539-3p could inhibit tumor growth in vivo. These findings suggest that miR-539-3p/CDK14 can serve as a potential therapeutic target for colon cancer.

\section{Acknowledgments}

Funding: None.

\section{Footnote}

Reporting Checklist: The authors have completed the ARRIVE reporting checklist. Available at http://dx.doi. org/10.21037/jgo-20-387

Data Sharing Statement: Available at http://dx.doi. org/10.21037/jgo-20-387

Conflicts of Interest: All authors have completed the ICMJE uniform disclosure form (available at http://dx.doi. org/10.21037/jgo-20-387). The authors have no conflicts of interest to declare.

Ethical Statement: The authors are accountable for all aspects of the work in ensuring that questions related to the accuracy or integrity of any part of the work are appropriately investigated and resolved. Animal experiments were conducted according to the National Institutes of Health (NIH) Guidelines for the Care and Use of Laboratory Animals and were approved by the Chengdu 
Fifth People's Hospital.

Open Access Statement: This is an Open Access article distributed in accordance with the Creative Commons Attribution-NonCommercial-NoDerivs 4.0 International License (CC BY-NC-ND 4.0), which permits the noncommercial replication and distribution of the article with the strict proviso that no changes or edits are made and the original work is properly cited (including links to both the formal publication through the relevant DOI and the license). See: https://creativecommons.org/licenses/by-nc-nd/4.0/.

\section{References}

1. Hodgkinson N, Kruger CA, Abrahamse H. Targeted photodynamic therapy as potential treatment modality for the eradication of colon cancer and colon cancer stem cells. Tumour Biol 2017;39:1010428317734691.

2. Roth AD, Tejpar S, Delorenzi M, et al. Prognostic role of KRAS and BRAF in stage II and III resected colon cancer: results of the translational study on the PETACC-3, EORTC 40993, SAKK 60-00 trial. J Clin Oncol 2010;28:466-74.

3. Wardhani Y, Hutajulu SH, Ferianti VW, et al. Effects of oxaliplatin-containing adjuvant chemotherapy on shortterm survival of patients with colon cancer in Dr. Sardjito Hospital, Yogyakarta, Indonesia. J Gastrointest Oncol 2019;10:226-34.

4. Banerjee A, Pathak S, Subramanium VD, et al. Strategies for targeted drug delivery in treatment of colon cancer: current trends and future perspectives. Drug Discov Today 2017;22:1224-32.

5. Zhao Y, Hu X, Zuo X, et al. Chemopreventive effects of some popular phytochemicals on human colon cancer: a review. Food Funct 2018;9:4548-68.

6. Gregory RI, Chendrimada TP, Cooch N, et al. Human RISC couples microRNA biogenesis and posttranscriptional gene silencing. Cell 2005;123:631-40.

7. Hwang HW, Mendell JT. MicroRNAs in cell proliferation, cell death, and tumorigenesis. Br J Cancer 2006;94:776-80.

8. Volinia S, Calin GA, Liu CG, et al. A microRNA expression signature of human solid tumors defines cancer gene targets. Proc Natl Acad Sci U S A 2006;103:2257-61.

9. Guo J, Gong G, Zhang B. miR-539 acts as a tumor suppressor by targeting epidermal growth factor receptor in breast cancer. Sci Rep 2018;8:2073.

10. Zhang H, Li S, Yang X, et al. miR-539 inhibits prostate cancer progression by directly targeting SPAG5.J Exp
Clin Cancer Res 2016;35:60.

11. Jin W, Han H, Liu D. Downregulation miR-539 is associated with poor prognosis of gastric cancer patients and aggressive progression of gastric cancer cells. Cancer Biomark 2019;26:183-91.

12. Yu H, Gao G, Cai J, et al. MiR-539 functions as a tumor suppressor in pancreatic cancer by targeting TWIST1. Exp Mol Pathol 2019;108:143-9.

13. Zhou J, Su M, Zhang H, et al. miR-539-3P inhibits proliferation and invasion of gastric cancer cells by targeting CTBP1. Int J Clin Exp Pathol 2019;12:1618-25.

14. Gong YB, Fan XH. MiR-539-3p promotes the progression of epithelial ovarian cancer by targeting SPARCL1. Eur Rev Med Pharmacol Sci 2019;23:2366-73.

15. Wen D, Li S, Jiang W, et al. miR-539 inhibits human colorectal cancer progression by targeting RUNX2. Biomed Pharmacother 2017;95:1314-20.

16. Sherr CJ, Roberts JM. Living with or without cyclins and cyclin-dependent kinases. Genes Dev 2004;18:2699-711.

17. Yang T, Chen JY. Identification and cellular localization of human PFTAIRE1. Gene 2001;267:165-72.

18. Ou-Yang J, Huang LH, Sun XX. Cyclin-Dependent Kinase 14 Promotes Cell Proliferation, Migration and Invasion in Ovarian Cancer by Inhibiting Wnt Signaling Pathway. Gynecol Obstet Invest 2017;82:230-9.

19. Imawari $Y$, Mimoto R, Hirooka $S$, et al. Downregulation of dual-specificity tyrosine-regulated kinase 2 promotes tumor cell proliferation and invasion by enhancing cyclindependent kinase 14 expression in breast cancer. Cancer Sci 2018;109:363-72.

20. Chen L, Wang Y, Jiang W, et al. CDK14 involvement in proliferation migration and invasion of esophageal cancer. Ann Transl Med 2019;7:681.

21. Li J, Shao W, Feng H. MiR-542-3p, a microRNA targeting CDK14, suppresses cell proliferation, invasiveness, and tumorigenesis of epithelial ovarian cancer. Biomed Pharmacother 2019;110:850-6.

22. Zhou Y, Rideout WM 3rd, Bressel A, et al. Spontaneous genomic alterations in a chimeric model of colorectal cancer enable metastasis and guide effective combinatorial therapy. PLoS One 2014;9:e105886.

23. Ji Q, Xu X, Xu Y, et al. miR-105/Runx2 axis mediates FGF2-induced ADAMTS expression in osteoarthritis cartilage. J Mol Med (Berl) 2016;94:681-94.

24. Zhu J, Liu C, Liu F, et al. Knockdown of PFTAIRE Protein Kinase 1 (PFTK1) Inhibits Proliferation, Invasion, and EMT in Colon Cancer Cells. Oncol Res 2016;24:137-44. 
25. Yoon H, Kim M, Jang K, et al. p27 transcriptionally coregulates cJun to drive programs of tumor progression. Proc Natl Acad Sci U S A 2019;116:7005-14.

26. Li N, Zeng J, Sun F, et al. p27 inhibits CDK6/CCND1 complex formation resulting in cell cycle arrest and inhibition of cell proliferation. Cell Cycle 2018;17:2335-48.

27. Bu H, Liu D, Cui J, et al. Wnt/ $\beta$-catenin signaling pathway is involved in induction of apoptosis by oridonin in colon cancer COLO205 cells. Transl Cancer Res 2019;8:1782-94.

28. Dong Y, Sun Y, Huang Y, et al. Depletion of MLKL inhibits invasion of radioresistant nasopharyngeal carcinoma cells by suppressing epithelial-mesenchymal transition. Ann Transl Med 2019;7:741.

29. Canel M, Serrels A, Frame MC, et al. E-cadherin-integrin crosstalk in cancer invasion and metastasis. J Cell Sci 2013;126:393-401.

30. Mendonsa AM, Na TY, Gumbiner BM. E-cadherin in contact inhibition and cancer. Oncogene 2018;37:4769-80.

31. Derycke LD, Bracke ME. N-cadherin in the spotlight of cell-cell adhesion, differentiation, embryogenesis, invasion and signalling. Int J Dev Biol 2004;48:463-76.

32. Cao ZQ, Wang Z, Leng P. Aberrant N-cadherin expression in cancer. Biomed Pharmacother 2019;118:109320.

33. Lin S, Gregory RI. MicroRNA biogenesis pathways in cancer. Nat Rev Cancer 2015;15:321-33.

34. Nana-Sinkam SP, Croce CM. MicroRNA regulation of tumorigenesis, cancer progression and interpatient heterogeneity: towards clinical use. Genome Biol 2014;15:445.

35. Su H, Wang X, Song J, et al. MicroRNA-539 inhibits the progression of Wilms' Tumor through downregulation of

Cite this article as: Wang Z, Hu T, Jin C, Yu J, Zhu D, Liu J. The anti-tumor effect of miR-539-3p on colon cancer via regulating cell viability, motility, and nude mouse tumorigenicity with CDK14 inhibition. J Gastrointest Oncol 2020;11(5):899-910. doi: 10.21037/jgo-20-387
JAG1 and Notch1/3. Cancer Biomark 2019;24:125-33.

36. Zhu C, Zhou R, Zhou Q, et al. microRNA-539 suppresses tumor growth and tumorigenesis and overcomes arsenic trioxide resistance in hepatocellular carcinoma. Life Sci 2016;166:34-40.

37. Mao Y, Jia Y, Zhu H, et al. High expression of PFTK1 in cancer cells predicts poor prognosis in colorectal cancer. Mol Med Rep 2017;16:224-30.

38. Yang L, Zhu J, Huang H, et al. PFTK1 Promotes Gastric Cancer Progression by Regulating Proliferation, Migration and Invasion. PLoS One 2015;10:e0140451.

39. Gu X, Wang Y, Wang H, et al. Upregulated PFTK1 promotes tumor cell proliferation, migration, and invasion in breast cancer. Med Oncol 2015;32:195.

40. Deng H, Qianqian G, Ting J, et al. miR-539 enhances chemosensitivity to cisplatin in non-small cell lung cancer by targeting DCLK1. Biomed Pharmacother 2018;106:1072-81.

41. Zhao J, Xu J, Zhang R. MicroRNA-539 inhibits colorectal cancer progression by directly targeting SOX4. Oncol Lett 2018;16:2693-700.

42. Yang J, Zhu H, Jin Y, et al. MiR-431 inhibits cell proliferation and induces cell apoptosis by targeting CDK14 in pancreatic cancer. Eur Rev Med Pharmacol Sci 2018;22:4493-9.

43. Li Q, Zhou L, Wang M, et al. MicroRNA-613 impedes the proliferation and invasion of glioma cells by targeting cyclin-dependent kinase 14. Biomed Pharmacother 2018;98:636-42.

(English Language Editor: A. Kassem) 